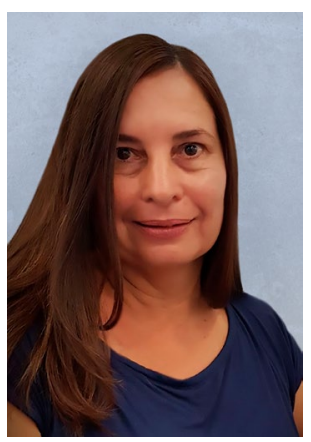

\title{
From denial to hope: Brazil deals with a prolonged COVID-19 epidemic course
}

\author{
Brazil must strengthen science-based education to improve human development and \\ responses to future public health threats.
}

Credit: Márcio Alves

A $t$ the end of February 2020, when I apprehensively followed the advance of a deadly wave of COVID-19 in Europe, huge crowds clustered in the streets to celebrate Carnival in Brazil. Later, epidemiologists revealed that SARS-CoV-2 had reached Brazil 1-3 months before the first case of COVID-19 was reported on 25 February 2020.

In the previous five years, Brazilians had struggled with an economic recession coupled with sharp political and social tensions, further fueled by science-negating leaders. Moreover, the Brazilian government has disrupted the environmental protection agencies, allowing the upscaling of illegal forest clearing, which accelerates climate change and the emergence of new zoonotic diseases. In the Amazon rainforest, the indigenous populations have the highest risk of poor social and health outcomes. Undoubtedly, this alarming scenario precludes the necessary efforts to cope with the COVID-19 pandemic and compromises the response to future public health and environmental crises in Brazil.

The dismantling of a dedicated team at the Ministry of Health, caused by the exclusion of experienced epidemiologists, prevented the establishment of a national leadership group and coherent public health policies to confront COVID-19. After the first reported death on 16 March, scientific boards recommended mobility and other restrictions in the metropolitan areas, which were also implemented around the country, at the discretion of local authorities. The infection reproduction rate $\left(R_{0}\right)$ abruptly dropped from 3 (de Souza, W. M. et al. Nat. Hum. Behav. https://doi. org/10.1038/s41562-020-0928-4, 2020) to slightly above 1 , and it has remained at that level since then. Likewise, there has been continuous reporting of deaths, rather than in successive waves, which has been a hallmark of COVID-19 in Brazil, caused by SARS-CoV-2 propagation and recirculation throughout the Brazilian states. At the end of January 2021, Brazil had already lost over 220,000 lives, and there had been more than 9 million cases of infection.

Exposed to conflicting information from the news and different layers of government, and overshadowed by misinformation from social media, Brazilians were left on their own to make decisions about life-saving measures, such as wearing masks and adopting social distancing. Hence, as the antivaccine movements resurged, the number of people receiving routine vaccines has fallen in Brazil, despite the previous successful immunization programs. Furthermore, recent surveys revealed an ongoing campaign against SARS-CoV-2 vaccines, whereas current political rivalries may ultimately hamper their management and distribution. Thus, while vaccination becomes a reality in developed countries, it remains an unfulfilled promise here.

After a decade of progress, recent Brazilian governments have substantially decreased investment in science, compromising the development of human and material resources. Postdocs have been particularly affected, by joining and thus expanding large research groups with available fellowships, by moving abroad for opportunities, or by leaving their scientific careers altogether. Moreover, postdocs do not have opportunities to develop independent careers, as jobs remain scarce at our universities. The uncertainty about work conditions as well as the quarantine certainly heightened anxiety levels, mainly among graduate students, and overwhelmed female scientists with children who required home care.

During social isolation that was imposed to mitigate the COVID-19 pandemic, most scientists at the universities were unprepared for virtual teaching, and they had to learn new strategies to interact with graduate and undergraduate students. In parallel, we have experienced new and exciting national and international webinars.
This outstanding intellectual network has kept young scientists enthusiastic and has compensated the community for the lack of scientific meetings.

This year, government agencies concentrated resources and fellowships on COVID-19 efforts. Brazilian scientists promptly responded with valuable research into the pathogenic roles of neutrophil extracellular traps (Veras, F. P. et al. J. Exp. Med. 217, e20201129, 2020) and inflammasomes (Rodrigues, T. S. et al. J. Exp. Med. 218, e20201707, 2020), providing new avenues for therapy. Biochemists and immunologists at my university cooperated to develop inexpensive diagnosis tools and hyperimmune sera in partnership with the Vital Brazil Institute. The Oswaldo Cruz and Butantan Institutes, universities and pharmaceutical companies assembled international networks to conduct investigations and clinical trials on immunotherapy and vaccine development.

While the level 3 biosafety labs, which can run SARS-CoV-2 experiments, became busier, most laboratories remained closed for 2-3 months, and essential services such as animal and equipment facilities have run under restrictions. Since July, following the stepwise reopening of the economy, research has gradually returned with restricted numbers and rotating lab personnel. Nevertheless, viral infection has been detected in the university campuses, while simultaneously, we have seen a steady increase in the cases of COVID-19 and overloaded hospitals, leading to some tragic outcomes. Moreover, a rise in rates of respiratory syndrome and insufficient testing point to underreporting of SARS-CoV-2 in the country.

Epidemiological surveys indicate that the prevalence of infection inversely correlates with educational, economic and social indicators. Sadly, SARS-CoV-2 infection disseminates in the neighborhoods where big families share small houses and use overcrowded public transportation to travel 
to workplaces and schools. More recently, infection has spread in crowded shopping areas, beaches, bars and parties, reflecting denial, as well as little responsibility, empathy, solidarity or respect for patients, mourning families and healthcare workers.

Overall, the advent of COVID-19 has strengthened our view that social and educational issues underlie the risk of exposure to life-threatening infectious diseases and that lack of knowledge is one of the saddest outcomes of social inequity. In this regard, it urges us to educate Brazilian children in the principles of science, such as ethics, critical thinking and searching for reliable information.

Fortunately, COVID-19 has placed science and immunology in the headlines, with the Brazilian media highlighting the unprecedented vaccine endeavor. By teaching graduate students engaged in our successful programs about immunology as a scientific career, I have realized that we do need more scientists to overcome COVID-19 and future public health challenges. I hope that increasing social inclusion of young people through education and science will help human and scientific development and reduce the economic and social gaps between Brazil and developed countries.

\section{Marcela F. Lopes (D)}

Carlos Chagas Filho Institute of Biophysics, Federal University of Rio de Janeiro, Rio de Janeiro, Brazil.

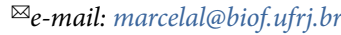

Published online: 24 February 2021

https://doi.org/10.1038/s41590-021-00875-8

Competing interests

The author declares no competing interests. 